\title{
Caring for elderly patients with dementia: nursing interventions
}

This article was published in the following Dove Press journal:

Nursing: Research and Reviews

5 August 2013

Number of times this article has been viewed

\author{
Laura L Joosse' \\ Debra Palmer' \\ Norma M Lang ${ }^{2}$ \\ 'University of Wisconsin-Milwaukee, \\ College of Nursing, Milwaukee, \\ WI, USA; ${ }^{2}$ University of Wisconsin- \\ Milwaukee, College of Nursing, \\ Knowledge Based Nursing Research \\ Initiative, Milwaukee, WI, USA
}

\begin{abstract}
Elderly patients suffering from chronic cognitive decline/dementia are susceptible to poor quality of care which further erodes their quality of life. Seemingly benign events can create cascade iatrogenesis in those whose compensatory ability is compromised by impairments in multiple domains. Under recognition, misrecognition, or failure to intervene and manage this vulnerable population leads to suboptimal care. This places them at risk for cognitive decline, functional decline, and challenging behaviors, creating financial and emotional burdens for not only the patients but also family, staff, and organizations that are attempting to provide care. Identifying, managing, and therapeutically responding to confused elderly is complex. Recognizing the challenges makes the development of tools that guide comprehensive assessment planning, interpretation of findings, and treatment plans imperative. Innovative and effective assessment and interventional approaches are present in the literature. This article synthesizes the scientific evidence to guide clinicians to implement in practice.
\end{abstract}

Keywords: dementia, older adults, assessment, intervention, quality of life, elderly, cognitive decline

\section{Introduction}

Hospitalized patients suffering from the sequelae of dementia have complex needs which challenge the delivery of acute nursing care. Despite advances in care, these older adults struggle with progressive cognitive decline, functional decline, and challenging behaviors that impact their quality of life. Suboptimal health care further erodes their quality of life. This is a prominent and growing health problem that impacts patients, families, health care systems, and society. These patients are at risk for inadequate care due to health care providers' lack of understanding of the etiology of cognitive impairment and misperception of the problem. ${ }^{1}$ This article aims to describe: (1) challenges in providing care to hospitalized patients suffering from dementia and delirium superimposed on dementia, (2) nursing assessments, problem identification, and interventions for dementia and delirium that may promote quality of life and care, and (3) synthesized recommendations to guide clinical practice.

Cognitive impairment can be categorized into two broad groups: chronic cognitive decline (diagnosable dementia) or acute cognitive changes (most likely the result of delirium). Accordingly, timely and accurate identification of the impairment/problem is important for several reasons. Under recognition, misrecognition, or failure to intervene and manage this vulnerable population leads to suboptimal care. Cascade iatrogenesis may be started by seemingly benign events in those whose compensatory ability is compromised by impairments in multiple domains. In addition, acute
Correspondence: Laura L Joosse University of Wisconsin-Milwaukee, College of Nursing, PO Box 413, Milwaukee, WI 5320I-04I3, USA

$\mathrm{Tel}+\mathrm{I} 4142296920$

Fax +I 4I4 2296474

Email lljoosse@uwm.edu 
changes in cognition (delirium) may be an early warning of the presence of systemic illness. ${ }^{1}$ Identifying, managing, and therapeutically responding to cognitive status and changes in an older adult population is a complex endeavor. The difficulty in recognizing the challenges makes it imperative that the best known evidence is readily available to nurses to guide assessments, interpretation of findings, and intervention/ treatment plan creation that lead to improvements in quality of life. Hospitalization of patients with dementia adds to the complexity and challenge of treatment; the care focus is on the acute medical surgical reasons (chief complaint) for the admission. Cognitive impairment, dementia, and delirium are often overlooked in the diagnosis and treatment plans. The advent of electronic medical records further complicates adequate information transfer with the need to develop electronic readable data that describes dementia and delirium and that can be included in clinical information systems and decision support.

\section{Background and significance}

Providing optimal care for older adult populations with cognitive decline is an increasing concern for health professionals and policymakers as evidenced by the passing of the One Hundred Eleventh Congress of the United States of America "National Alzheimer's Project Act."'2 Recommended priorities described in the bill include, but are not limited to, actions that improve prevention, treatment, and care for those receiving services in health care institutions focusing on quality and cost effectiveness. This is of significant importance because it is estimated that by the year 2030 there will be 65.7 million people with dementia worldwide ${ }^{3}$ with approximately $17 \%$ spending the end stages of their lives in nursing homes and many needing repeated hospitalizations. ${ }^{4,5}$

Alzheimer's disease, other related dementias, and delirium are common phenomena causing cognitive decline with similar and overlapping features. Overlapping symptoms make it difficult to ascertain baseline mental status and the acuteness of the symptoms. ${ }^{6-8}$ Some older adults may have undetected dementia because slow progressive cognitive changes may not be recognizable while in a familiar environment. However, changes may become apparent when the patient enters an unfamiliar environment such as the hospital. Kennedy has observed that patient families will report "my mother was never like this at home" suggesting a change has occurred during or as the result of the transition of care. ${ }^{9}$

Overall, recognition of states of confusion is problematic. In a study of focus group interviews of 23 general practitioners
(GPs) from different disciplines, reluctance in confirming a diagnosis of dementia due to social stigma and feelings of having little to no treatment options in the early stages of the disease was admitted. ${ }^{10}$ Boustani et al found that while $43 \%$ of their sampled population $(\mathrm{N}=997)$ scored positively for cognitive impairments, only 424 (61\%) of those with cognitive impairment had documentation of the presence of cognitive deficits in the electronic medical record at discharge. ${ }^{11}$ Similarly, in a pilot study of 145 patients ( 65 years or older) admitted to three hospitals, 51 patients (35\%) had cognitive impairment consistent with dementia. Yet, there was no documentation related to signs of dementia in 20 (39\%) of the patient records. ${ }^{12}$ These studies are further supported by the work of Mitchell et al. ${ }^{13}$ These authors ${ }^{13}$ conducted a metaanalysis from an extensive review of the recent literature and concluded that GPs recognized mild cognitive impairment only $44.7 \%$ of the time and documented this in the patient's medical record only $10.9 \%$ of the time.

Delirium superimposed on dementia adds another layer to the problem. In a seminal integrated literature review, Fick et al estimated that the prevalence of delirium superimposed on dementia ranged from $22 \%-89 \%$ in hospitalized and community populations. ${ }^{14}$ Accordingly, the majority of the studies reported ${ }^{14}$ greater than $50 \%$ prevalence of delirium with rates as high as $89 \%$ in people with dementia. In addition, several studies hypothesized that professionals are less likely to recognize insidious forms of delirium. The review found the frequent lack of recognition, the lack of preventative strategies with behavioral approaches, and the use of psychoactive drugs were problematic for this subgroup of patients. ${ }^{14}$

The insufficient detection of cognitive impairment, among hospitalized older adults, places the elderly at risk for suboptimal care. ${ }^{15}$ Hospitalized people with chronic cognitive decline are at risk for falling, impaired gait and balance, inattention, orthostatic hypotension, eating/ hydration problems, inadequate rest/sleep, untreated pain, and becoming overwhelmed by being in an unfamiliar environment. ${ }^{16}$ They are also at risk for delirium from medication side effects, including pain medications and treatment of acute illness. In addition, they are more likely to exhibit challenging behaviors (wandering, agitation) and may experience functional decline. Best practice approaches aimed at controlling risk factors, promoting independence, and strategies to prevent behavioral consequences and functional decline are available. ${ }^{8,17-19}$ However, gaps remain in the best way to synthesize and translate the evidence to the bedside caregiver. 
The clinical management of patients with dementia is problematic due to the frequent presence of behavioral and psychological co-occurring symptoms. ${ }^{20}$ Research has demonstrated that cognitive impairments in the elderly patient interfere with nursing care. In a study of 133 caregivers, nurses identified that cognitive symptoms interfered with care (memory $96 \%$, lack of insight/judgment $94 \%$, loss of purposeful movement 92\%). ${ }^{21}$ However, those who identified a cognitive symptom as interfering with care had difficulty indicating a strategy to minimize that impact (memory $50 \%$, lack of insight/judgment $60 \%$, loss of purposeful movement 46\%). ${ }^{21}$ This study highlights issues beyond just recognizing the problem. The nurses in this study recognized the interference with care but $40 \%-50 \%$ were not able to strategize interventions that might make a difference. Nurses need tools that will not only guide assessment but provide a framework to choose evidenced based interventions and treatment plans as well.

Functional impairment and behavioral symptoms are common consequences of confusion caused by delirium or dementia. ${ }^{22}$ They include dependence in daily activities, inability to initiate meaningful activity, spatial disorientation, and anxiety, which can lead to agitation, apathy, insomnia, repetitive vocalization, resistiveness, combativeness, food refusal, elopement/wandering, and interference with care. Functional decline and behavioral changes are also associated with acute hospitalization. Early assessment and preventative measures may be useful in preserving function and preventing further decline saving health care dollars by avoiding suboptimal care..$^{22,23}$

In a well established evidence based geriatric nursing protocol, assessment of symptomatic individuals is recommended to alert clinicians to the extent of the cognitive and functional decline. ${ }^{23}$ The goal for assessment of cognitive impairment is to help in differentiating dementia from delirium and/or depression. Each condition requires tailored interventions based on the cause and presentation. This study highlights that some nurses may fail to recognize cognitive impairment, while others recognize but are not sure how to address the problem they have identified. Bringing evidenced based assessment and intervention practice recommendations to the bedside holds great promise for improving care and quality of life for these complex patients.

One way to bring the best evidence to the bedside for the nurse is to embed the evidence in electronic information systems as clinical decision support (CDS). Within the complexities of the patients' and the nurses' workflow, specific memory based care may add to the burden. It is not feasible to expect nurses to readily recall the battery of assessments, key risk factors that trigger the problem, linkages to appropriate nursing specific interventions, or to go outside of the workflow to find the resources that help find the answers and guide their practice.

Some authors recommend a patient safety paradigm that focuses on implementing evidence based practice (EBP). ${ }^{24}$ The recommendation concludes that when EBP is followed, patients will receive more appropriate care leading to better quality of care. No single reference can provide all the evidence based practice information necessary to care for the older adult. The key is to bring a comprehensive EBP framework of information to the bedside for frontline caregivers to integrate into their practice. CDS solutions are one proposed method of translating EBP knowledge. ${ }^{25-28}$

The findings of this review provide evidence that can support bedside clinical decision making and may be used in the design and build of electronic support systems. Bakken et al assert that stand alone approaches that strive to assist nurses in accessing evidence necessary for practice are inadequate. ${ }^{29}$ They recommend integrating the information needed by nurses into the workflow. Clinical information systems, specifically CDS solutions can be used to support the process of knowledge translation at the bedside for frontline nurses. CDS solutions provide clinical support for care strategies that promote evidenced based early recognition, risk assessment, problem identification, and selection of individualized interventions, and outcomes. ${ }^{27}$

\section{Conceptual definitions}

For this literature review, we were interested in capturing data variables for nursing assessment, problem identification, and nursing interventions for the hospitalized patient with dementia. These variables are defined at the conceptual level within the framework of the nursing process. The nursing process provides a problem solving approach to the identification and treatment of patient health and illness problems. ${ }^{30}$ Hence, these nursing process variables guided the criteria for selecting studies and facilitating data elements. The nursing process variables are defined as follows: (1) nursing assessment is the first phase of the nursing process where information is collected based on subjective and objective patient data that is necessary for identifying accurate diagnoses, planning, and interventions; ${ }^{30}(2)$ problem identification/nursing diagnosis phase begins with clustering information and ends with a judgment about the patient's health problem; ${ }^{30}$ and (3) nursing intervention is a treatment that nurses perform in relation to the assessments to enhance patient outcomes. ${ }^{30}$ 


\section{Methods}

An integrated literature review was performed to identify the best known evidence on the topic of dementia (chronic cognitive decline) for older hospitalized adults. The nursing process components were used as the framework, identifying key evidence that guides nursing assessment, diagnosis, and interventions. Hong reported the method for topic/question formulation and use of these nursing process components. ${ }^{31}$ This method included guiding questions for the topic, type of evidence, findings related to background, significance of the problem, findings related to patient assessment, nursing diagnosis, and interventions. ${ }^{31}$ This article is limited to the synthesis and recommendations for the topic of dementia including components of assessment, problem identification, and interventions focusing on promoting quality of life.

Prior to the search, a group of methods and content experts from both academia and hospital operations met to define the phenomenon of concern and search terms. Next, an expert librarian formulated a search strategy and began the iterative process of extracting articles that matched the phenomenon of concern to describe recommended concepts of the nursing process. Electronic databases were searched using the following criteria: English language text published between 2002 and 2012, including additional seminal dated articles outside the 10 year framework. The literature search focused on nursing assessment, nursing diagnoses, and nursing interventions. Venue focus included hospitalized medical and surgical patients and elements that were nonvenue specific but pertinent and important to include. Intensive care unit patient literature was excluded. Searches were limited to meta-analyses, systematic reviews, clinical trials, professional guidelines, standards of care, and articles from peer reviewed journals and professional texts.

Key terms were selected based on input from four content experts and an expert in meta-analysis. Ninety-nine major search terms/phrases alone and in various combinations as subject headings were used. An example of some of the key terms used included dementia, confusion, chronic cognitive decline, disorientation, sundown syndrome, Alzheimer's disease, cognitive impairment, and cognitive disorders. Databases searched using the select keywords or phrases included PubMed, Cumulative Index to Nursing and Allied Health Literature, PsychInfo, Embase, Cochrane Database of Systematic Reviews, Mosby's Nursing Consult, Health and Psychosocial Instruments, Allied and Complementary Medicine Database, and Thomson Reuters Web of Science.

\section{Sample}

Six hundred and ten abstracts were reviewed for content and 325 were selected based on question guidance. ${ }^{31}$ The selected articles were reviewed and sorted. Those included met the criteria of hospitalized patients or nonvenue focus. Next, using the Melnyk and Fineout-Overholt rating system for hierarchy of evidence, the articles were rated. ${ }^{32}$ Articles rated level 1 to level 8 were included. Articles not meeting question guidance, level of evidence criteria, or venue focus were excluded. Sixty sources were selected and included in this review.

Each study, article, or text literature was rated on the level of evidence. In addition, the researchers analyzed several other factors during the process of the literature review including (1) degree of similarity of findings between studies, (2) research setting and sample, (3) clinically important recommendations for practice, and (4) how the findings of the studies may be translated into the practice environment. The researchers evaluated the retrieved evidence for validity, relevance, and application to the hospital environment, synthesized the best available evidence, and made recommendations for each of the nursing process components. Once recommendations were made, the synthesis was evaluated through an evidence review by three well established experts in clinical practice, meta-analysis, and synthesis consultation.

\section{Findings}

Sixty publications met the inclusion criteria for this review. These sources included EBP guidelines $(\mathrm{N}=9)$, clinical education articles $(\mathrm{N}=24)$, descriptive studies $(\mathrm{N}=13)$, systematic literature reviews $(\mathrm{N}=8)$, and psychometric studies $(\mathrm{N}=6)$. Findings from the 60 sources of evidence identified 59 nursing process components that are recommended. Table 1 provides an overview of the recommendations. The nursing assessment factors identified intended to provide an algorithmic way to identify patients 65 years or older on admission with the presence of symptoms, behaviors, or history of cognitive impairment consistent with dementia while differentiating from delirium and offering specific strategies to prevent further decline of cognition, function, and behavioral symptoms.

Based on the literature, it is recommended to screen individuals 65 years or older, on admission, for history or presence of cognitive decline. For patients with a known history of chronic cognitive decline, additional information should be obtained from the medical record, patient, family, caregiver, or other sources available. The data collected should include symptoms or behaviors including type of symptoms, patterns, course, onset, and duration. For those who do not 
Table I Recommendation overview

Assessment recommendations

I. For patients 65 years or older, on admission, screen for:

a. History or diagnosis of dementia or chronic cognitive decline

b. History of cognitive impairment

c. For patient with a known history of dementia/chronic cognitive decline or other cognitive impairment, gather additional details about dementia or chronic cognitive decline (based on medical record, information from patient, family, caregiver, or other sources as available)

2. For patients 65 years or older who do not have an existing diagnosis of delirium, dementia, or chronic cognitive decline, on admission screen for signs and symptoms of confusion (mental status disturbances and/or fluctuations in mental status)

For patients with signs and symptoms of confusion (mental status disturbances and/or fluctuations in mental status), determine whether the confusion is of:

a. Acute/rapid onset, within a short time frame, and fluctuating in course or

b. Chronic, with an overall pattern of decline over time, based on the following assessments from patient or family/caregiver report, or other sources as available

3. For patients with signs and symptoms of chronic cognitive decline, assess for cognitive impairment using a valid and reliable tool, if available

4. For patients with signs and symptoms of chronic cognitive decline, assess for limitations in ability to complete activities of daily living (ADL) including bathing, dressing, toileting, transferring to bed or chair, continence, and eating, from self-report or family/caregiver report

Problem identification recommendation

I. Chronic cognitive decline/dementia

Intervention recommendations

I. For patients with chronic cognitive decline or a diagnosis of dementia implement the following:

a. Therapeutic communication strategies

b. Strategies to reduce internal stressors

c. Strategies to reduce external stressors

d. Involve the family/caregiver in the plan of care when capable, willing, and appropriate

e. Strategies to promote patient safety

f. For patients with chronic cognitive decline who need assistance or who are dependent in ADL:

I. Implement the following based on needs

2. Monitor ADL daily

2. For individuals who are experiencing disruptive behaviors such as agitation, wandering, or resistiveness to care:

a. Provide nonpharmacological interventions first

b. Collaborate with the physician regarding pharmacological interventions if nonpharmacological interventions are not successful

c. If the patient is severely distressed or if there is an immediate risk of harm to the person or others, pharmacological interventions should be implemented first

3. For patients who have chronic cognitive decline, assess for changes in mental status approximately every $8-12$ hours for acute confusion (delirium) using a valid reliable tool where available:

a. Cognition

b. Behavior

c. Fluctuation in mental status

d. Altered sleep-wake cycle

e. Psychomotor behavior

4. For patients who do not have an existing diagnosis of chronic cognitive decline or dementia, but who screen positive for cognitive impairment, collaborate with the primary care provider to determine if a diagnostic interview based on the DSM-IV criteria for dementia is warranted

Abbreviation: DSM-IV, Diagnostic and Statistical Manual of Mental Disorders -IV.

have a history of dementia or delirium, the screening should include symptoms of mental status disturbances and/or fluctuations in mental status. The screening determines whether the confusion is acute, indicating delirium, or chronic, suggesting dementia. Table 2 provides these specific assessment recommendations identified from the literature, including three valid and reliable tools. In addition, assessment criteria include recommendations for evaluating activities of daily living (ADL) that directly impact the negative sequelae of acute hospitalization of our older adults. Overall, the evaluation of the assessment data can guide the selection of an intervention plan.
Table 3 provides an overview of best known evidence on interventional approaches. These intervention factors provide guidance in the prevention and management of further cognitive decline, ADL function, and behavioral symptoms that may be associated with hospitalization in those suffering from dementia. We grouped like interventions into categorized bundles. Intervention bundles include therapeutic communication methods, strategies to reduce internal and external stressors, strategies to involve family or caregivers in the plan of care, patient safety strategies, and ADL recommendations. Similarly, specific recommendations for managing patients who are exhibiting disruptive behaviors 
Table 2 Nursing assessment recommendations identified from the literature ${ }^{6,7,9,11}, 12,14,15,17-19,22,23,25,33-35,37,39,41-52,68,69,75,76$.

\section{Nursing assessment and factors}

For patients 65 years or older, on admission, screen for:

I. History or diagnosis of dementia or chronic cognitive decline

2. History of cognitive impairment

3. For patients with a known history of dementia/chronic cognitive decline or other cognitive impairment, gather additional details about dementia or chronic cognitive decline (based on medical record, information from patient, family, caregiver, or other sources as available):

a. Symptoms/behaviors

i. Symptom - types of symptoms present

ii. Patterns/frequency - how often and under what circumstances do symptoms occur?

iii. Course - how has it changed over time?

b. Onset - when did it start?

c. Duration - how long has it lasted?

For patients 65 years or older who do not have an existing diagnosis of delirium, dementia, or chronic cognitive decline, on admission screen for signs and symptoms of confusion (mental status disturbances and/or fluctuations in mental status)

For patients with signs and symptoms of confusion (mental status disturbances and/or fluctuations in mental status), determine whether the confusion is of:

I. Acute/rapid onset, within a short time frame, and fluctuating in course, OR

2. Chronic, with an overall pattern of decline over time, based on the following assessments from patient or family/caregiver report, or other sources as available

3. By assessing for:

a. Level of consciousness

b. Thought process

c. Attention

d. Orientation

e. Memory

f. Perception (hallucinations/illusions)

g. Sleep/wake cycle

h. Motor behavior

i. Affect/behavior

i. Verbal/physical aggression/agitation

ii. Resistance to care

iii. Wandering

Note: Acute change or fluctuating pattern of altered attention, thought process, and/or level of consciousness may indicate acute confusion (delirium)

For patients with signs and symptoms of chronic cognitive decline, assess for cognitive impairment using a valid and reliable tool, if available. The following tools are valid and reliable

I. Mini-Cog

2. Orientation-Memory-Concentration Test (short version Blessed Test)

3. Folstein's Mini-Mental State Examination (MMSE)

For patients with signs and symptoms of chronic cognitive decline, assess for limitations in ability to complete activities of daily living including bathing, dressing, toileting, transferring to bed or chair, continence, and eating, from self-report or family/caregiver report

are provided. Nonpharmacological interventions are recommended as a first line of management unless patients are at risk for harming themselves or others. The recommendations include strategies to manage agitation, wandering, and potential triggers to the behavior. Finally, recommendations to evaluate changes in mental status providing an algorithmic way for ongoing assessment to differentiate chronic from acute confusion states are provided.

\section{Discussion}

Bringing evidence to the bedside nurse is one way to promote quality of care to hospitalized patients suffering from the consequences of dementia. Stand alone approaches limit a nurse's ability to access information while providing care within task based systems. ${ }^{27}$ Current best practice knowledge is available but may not be easily accessible to the bedside nurse. As others have reported, CDS tools that embed the best known knowledge in an easily accessible format for the nurse offer a solution to this plaguing problem. ${ }^{27-29}$ Similarly, based on Lang's vision for the transformation of practice and research, within an era of electronic health records and clinical information systems environment, the work presented in this article represents the first component necessary to fill this gap. ${ }^{27}$

In this review, findings from the evidence that met the selection criteria were synthesized into recommendations 
Table 3 Nursing intervention recommendations identified from the literature

\section{Intervention recommendation and factors}

For patients with chronic cognitive decline or a diagnosis of dementia implement the following:

Therapeutic communication strategies

a. Establish person's primary language

b. Introduce self, offering day, date, time, and place during each interaction

c. Use patient's preferred first name

d. Use hearing and vision assistive devices as needed; ask family to bring from home

e. Approach patient in a calm, gentle and relaxed manner

f. Speak directly to the person (even if unable to respond) and make eye contact

g. Remain calm if patient is agitated

h. Avoid excess stimuli during communication

i. Allow ample time for patient to respond before repeating information

j. Limit choices

k. Observe both verbal and non-verbal methods of communication

I. Avoid moving or walking during communication

$\mathrm{m}$. Repeat sentences using the same words

n. Use short, simple sentences and a soft tone

o. Ask simple questions that require a short answer (yes/no)

Strategies to reduce internal stressors

a. Control pain

b. Provide adequate nutrition and fluids

c. Ambulate based on patients ability

d. Promote rest (avoid sleep medication)

e. Monitor side effects of medications

Strategies to reduce external stressors

a. Reduce environmental stimulation

I. Place patient in private room away from busy/noisy areas of the unit

b. Plan and maintain a consistent routine

c. Mimic home routine and rituals where possible

d. Provide memory aids such as calendars, schedules for the day

e. Provide consistent caregivers when possible

f. Provide diversion, such as music, games, repetitive hand activities (folding towels), reminiscence, and stuff animals/dolls

g. Avoid use of physical or pharmacological restraints

Involve the family/caregiver in the plan of care when capable, willing, and appropriate

a. Ask family to bring in pictures or familiar objects (eg, favorite hat)

b. Ask family member to stay (when possible) during times when behavioral changes are noticed

c. Include family members in providing care (ie, eating, bathing, toileting, walking)

Strategies to promote patient safety

a. Keep environment uncluttered and eliminate environmental hazards

b. Provide visual prompts and signage

c. Camouflage or hide intravenous catheters or other tubes, if in use For patients with chronic cognitive decline or a diagnosis of dementia who need assistance or are dependent in activities of daily living (ADL):

a. Implement the following based on needs:

I. Related to eating:

a. Provide eating assistive devices (covered cups, plate with suction)

b. Offer one food at a time

c. Cut food in small pieces

d. Offer finger foods

e. Feed when necessary
Table 3 (Continued)

2. Related to personal hygiene:

a. Cover bathroom mirror if image invokes fear or agitation

b. Bathe one part at time, keep body covered

c. Re-approach at a later time if care is refused

d. Toileting schedule

3. Related to impaired physical mobility

4. Monitor ability to perform ADL daily

a. For patients with a decline in ADL, collaborate with physician to evaluate the patient's status

For individuals who are experiencing disruptive behaviors such as agitation, wandering, or resistiveness to care:

a. Provide non-pharmacological interventions first

I. General strategies

a. Determine the potential source

i. Internal stressors

- Implement strategies to reduce internal stressors

ii. External stressors

- Implement strategies to reduce external stressors

b. Promote therapeutic communication

2. Strategies to manage agitated behavior

a. Diversion activities

i. Games, cards, stuff animals/dolls, folding towels

ii. Music and reminiscence therapy

b. Social contact

i. Encourage family member visitation

ii. One to one social interaction

iii. Volunteer visits

c. Environmental modifications

i. Manage stimulation

ii. Noise (provide periods of quiet time)

iii. Light (manage light to promote day/night cycle)

d. Examine potential triggers and patterns of behavior

3. Strategies to prevent and manage wandering behavior

a. Provide supervision/surveillance

i. Staff should accompany patient off the unit

ii. Place patient in a room that allows maximum surveillance

iii. Regular patient checks

iv. Volunteers or sitters when necessary

v. Bed/chair alarm

b. Reduce environmental triggers

i. Avoid rooms near high traffic or noise

ii. Keep stairs, elevators, and other exit cues out of the patient's view

iii. Keep suitcase, shoes, and street clothes out of the patient's view

iv. Position bed for best visibility and access to the bathroom

c. Provide individual interventions

i. Familiar objects

ii. Avoid room changes

iii. Reduce noise (provide periods of quiet time)

iv. Provide diversion therapy

v. Encourage movement and exercise (mobility)

vi. Assess and treat for pain

vii. Toilet and incontinence care

viii. Promote sleep/wake cycle

ix. Provide food and fluids 
Table 3 (Continued)

d. Strategies to manage resistiveness to care

i. Monitor and treat for pain

ii. Cover bathroom mirror if image invokes fear or agitation

iii. Allow patient to bath self when capable

iv. If requires assistance, bath one part at time, keep body covered

v. Re-approach at a later time if care is refused

vi. Use no-rinse disposable cloths

vii. Ask family to participate in care when appropriate

b. Collaborate with the physician regarding pharmacological interventions if non-pharmacological interventions are not successful.

c. If the patient is severely distressed or there is an immediate risk of

harm to the person or others, pharmacological interventions should be implemented first.

d. Consider implementing risk for falls protocol

For patients who have chronic cognitive decline, assess for changes in mental status approximately every 8-12 hours for acute confusion (delirium) using a valid reliable tool where available:

a. Cognition

I. Awareness

2. Alertness (level of consciousness)

3. Attention

4. Orientation

5. Memory

6. Thinking

7. Perception (Illusions, delusions, hallucinations)

b. Behavior

I. Verbal and/or physical aggression/agitation

2. Resistance to care

3. Wandering

c. Fluctuation in mental status

d. Altered sleep-wake cycle

e. Psychomotor behavior

For patients who do not have an existing diagnosis of chronic cognitive decline or dementia, but who screen positive for cognitive impairment, collaborate with the primary care provider to determine if a diagnostic interview based on the DSM-IV criteria for dementia is warranted. 14,18,20,25,36,38,39,53-74,76

Abbreviation: DSM-IV, Diagnostic and Statistical Manual of Mental Disorders -IV.

for assessment, problem identification, and interventions related to dementia in hospitalized older adults. This work represents the first two steps which include: (1) identifying, synthesizing, and rating the current nursing knowledge for the phenomena, and (2) making recommendations with discrete, clear data elements that are meaningful and ready for immediate use for clinical nurses, health information technology (HIT) developers, and researchers. ${ }^{27}$ This review provides the recommendations that are ready for the next steps in electronic decision support.

The next steps are the design and building of this best evidence into integrated HIT solutions. This information technology solution can also provide a Web based system that contains the database of articles used in the synthesis (referential knowledge). The practice recommendations are transformed into a machine readable format (actionable knowledge/data modeling) with standardized terminology to share data across a system. These coded data elements are embedded within an electronic "point of care" CDS and documentation system (executable knowledge). Once the electronic infrastructure is developed, the next step of the mission instills the synthesized knowledge into practice, where the nurses use the electronic clinical information system. The documented data are captured in a clinical repository and data warehouse where they can be retrieved and analyzed for reports, quality improvement studies, clinical research, and new knowledge discovery. This data set/base is created from numerous patient encounters and can also be used to study nursing practice, staffing, patient satisfaction, administrative data, and further research.

The steps described in this article are necessary to realize the full process of transforming the synthesized knowledge into actionable knowledge for use at the bedside. Having access to synthesized knowledge in an electronic format will speed up the vendor and health organization's electronic support systems providing nurses with access to the best available knowledge at the bedside. In sum, nurses are faced with complex patient situations and are challenged with making judgments integrating different aspects of data and information about the patient. Providing them with integrated HIT solutions, based on the best known evidence, provides patients with chronic cognitive decline the best chance of receiving optimal care during acute hospitalization. ${ }^{24-28}$ The synthesis and assessment, problem identification, and intervention recommendations identified in this article are the first step in this work.

\section{Conclusion}

In summary, the journey for those suffering from dementia should include supportive strategies for assessment, problem identification, and nursing interventions that aim to promote their quality of life which hinges on the knowledge of the patients cognitive state. Most emphasis for hospitalized patients is on the presenting medical/surgical problem and interventions. Mental and behavioral health problems and interventions, associated with dementia care, are often overlooked or receive little priority. ${ }^{15}$ Furthermore, the difficulty in identifying features of the mental and behavioral health conditions make it imperative that nurses recognize the problems through a comprehensive assessment, then link nursing interventions to the problem. ${ }^{33}$ Using knowledge through best available evidence offers hope that complex patients with dementia can receive 
optimal care. This review provides an overview of practice recommendations that are thought to promote quality of care, therefore promoting quality of life. The work is a first step toward an integrated coordinated plan of care for patients with medical, surgical and mental and behavioral health conditions. Also, the work provides foundational content for use in the design and build of complex electronic clinical information systems. We recognize that the importance of the content and decision support to clinicians must also be recognized appropriately in adequate staffing, competencies, productivity, and payment systems to be fully implemented.

\section{Disclosure}

The authors report no conflict of interest in this work.

\section{References}

1. Caplan J, Rabinowitz T. An approach to the patient with cognitive impairment: delirium and dementia. Med Clin North Am. 2010; 1103-1116.

2. One Hundred Eleventh Congress of the United States of America. National Alzheimer's Project Act. Washington: One Hundred Eleventh Congress of the United States of America; 2010. Available from: http://www.gpo.gov/fdsys/pkg/BILLS-111s3036enr/pdf/ BILLS-111s3036enr.pdf. Accessed July 7, 2013.

3. Dementia statistics [webpage on the Internet]. Alzheimer's Disease International. Available from: http://www.alz.co.uk/research/statistics. html. Accessed December 27, 2012.

4. Luppa M, Luck T, Brähler E, König HH, Riedel-Heller SG. Prediction of institutionalization in dementia. A systematic review. Dement Geriatr Cogn Disord. 2008;26(1):65-78.

5. Mitchell S, Teno J, Kiely D, et al. The clinical course of advanced dementia. New Engl J Med. 2009;361:1529-1538.

6. Arnold E. Sorting out the $3 \mathrm{D}$ 's: delirium, dementia, depression: learn how to sift through overlapping signs and symptoms so you can help improve an older patient's quality of life. Holis Nurs Pract. 2005;19(3):99-105.

7. Fick, D Mion L. Delirium superimposed on dementia. American Journal of Nursing. 2008;108(1):52-60.

8. Landers J, Bonner A. Evaluating and managing delirium, dementia, and depression in older adults hospitalized with otorhinolaryngic conditions. ORL Head Neck Nurs. 2007;25(3):14-25.

9. Kennedy GJ. Try this: best practices in nursing care for hospitalized older adults with dementia. Brief evaluation of executive dysfunction: an essential refinement in the assessment of cognitive impairment. Nebr Nurse. 2004;37(2):31.

10. Vernooij-Dassen MJ, Moniz-Cook ED, Woods RT, et al. Factors affecting timely recognition and diagnosis of dementia across Europe: from awareness to stigma. Int J Geriatr Psychiatry. 2005;20:377-386.

11. Boustani M, Baker MS, Campbell N, et al. Impact and recognition of cognitive impairment among hospitalized elders. J Hosp Med. 2010;5(2):69-75.

12. Naylor MD, Stephens C, Bowles KH, Bixby MB. Cognitively impaired older adults: from hospital to home. Am J Nurs. 2005;105(2):52-61.

13. Mitchell A, Meader N, Pentzek M. Clinical recognition of dementia and cognitive impairment in primary care: a meta-analysis of physician accuracy. Acta Psychiatr Scand. 2011;124:165-183.

14. Fick DM, Agostini JV, Inouye SK. Delirium superimposed on dementia: a systematic review. J Am Geriatr Soc. 2002;50(10):1723-1732.

15. Laurila JV, Pitkala KH, Strandberg TE, Tilvis RS. Detection and documentation of dementia and delirium in acute geriatric wards. Gen Hosp Psychiatry. 2004;26(1):31-35.
16. Kim D, Brown R, Ding E, Kiel D, Berry S. Dementia medications, and risk for falls, syncope, and related adverse events: Meta-analysis of randomized controlled trials. J Am Geriatr Soc. 2011;59:1019-1031.

17. Maslow K, Mezey M. Recognition of dementia in hospitalized older adults. Am J Nursing. 2008;108:40-49.

18. National Collaborating Centre for Mental Health. Dementia: A NICESCIE guideline on supporting people with dementia and their carers in health and social care. London; The British Psychological Society and Gaskell; 2007. Available from: http://www.nice.org.uk/nicemedia/ pdf/CG42Dementiafinal.pdf. Accessed February 1, 2010.

19. Registered Nurses Association of Ontario. Caregiving strategies for older adults with delirium, dementia and depression. Ontario: Registered Nurses Association of Ontario; 2004. Available from: http://rnao.ca/sites/rnao-ca/files/Caregiving_Strategies_for_Older_ Adults_with_Delirium_Dementia_and_Depression.pdf. Accessed May 3, 2011.

20. Brodaty H, Arasaratnam C. Meta-analysis of nonpharmacological interventions for neuropsychiatric symptoms of dementia. Am J Psychiatry. 2012;169(9):946-953.

21. McGilton K, Wells J, Davis A, et al. Rehabilitating patients with dementia who have had a hip fracture: Part II: Cognitive symptoms that influence care. Top Geriatr Rehabil. 2007;23(2):174-182.

22. Volicer L, Mckee A, Hewitt S. Dementia. Neurol Clin. 2001;19(40): $867-885$.

23. Fletcher K. Dementia. In: Capezuti E, Zwicker D, Mezey M, Fulmer T, Gray-Miceli D, editors. Evidence-based Geriatric Nursing Protocols for Best Practice. 3rd ed. New York, NY: Springer; 2008:83-109.

24. Brennan TA, Gawande A, Thomas E, Studdert D. Accidental deaths, saved lives, and improved quality. New Engl J Med. 2005;353(13): 1405-1409.

25. Ela S, Lang N, Lundeen S. Time for a nursing legacy: Ensuring excellence through actionable knowledge. Nurse Leader. 2006; $4(6): 42$.

26. Hook ML, Devine EC, Lang NM. Using a computerized fall risk assessment process to tailor interventions in acute care. (Vol 1: Assessment.) In: Henriksen K, Battles JB, Keyes MA, Grady ML, editors. Advances in Patient Safety: New Directions and Alternative Approaches. Rockville: Agency for Healthcare Research and Quality; 2008.

27. Lang NM. The promise of simultaneous transformation of practice and research with the use of clinical information systems. Nurs Outlook. 2008;56(5):232-236.

28. Lang NM, Hook ML, Akre ME, et al. Translating knowledge-based nursing into referential and executable applications in an intelligent clinical information system. In: Weaver C, Delaney C, Weber P, Carr R, editors. Nursing and Informatics for the 21st Century: An International Look at the Cases, Practice, and the Future. Chicago: Healthcare Information and Management Systems Society; 2006:291-303.

29. Bakken S, Currie LM, Lee NJ, Roberts WD, Collins SA, Cimino JJ. Integrating evidence into clinical information systems for nursing decision support. Int J Med Inform. 2008;77:413-420.

30. Lewis SL, Dirksen SR, Heitkemper MM, Bucher L, Camera I. MedicalSurgical Nursing: Assessment and Management of Clinical Problems. 8th ed. St Louis: Mosby; 2011.

31. Hong WS. Evidence-based nursing practice for health promotion in adults with hypertension: A literature review. Asian Nurs Res. 2010;4(4):227-245.

32. Melnyk BM, Fineout-Overholt E. Evidence-based Practice in Nursing and Healthcare: A Guide to Best Practice. Philadelphia, PA: Lippincott Williams \& Wilkins; 2010.

33. Persoon A, Banningh LJ, van de Vrie W, Olde Rikkert MG, van Achterberg T. Daily observation of cognitive functioning in hospitalized patients on acute geriatric wards. J Clin Nurs. 2009;18(13):1930-1936.

34. Beers MH, Berkow R, editors. The Merck Manual of Geriatrics. Whitehouse Station: Merck Research Laboratories; 2000

35. Ferri CP, Prince M, Brayne C, et al; Alzheimer's Disease International. Global prevalence of dementia: A Delphi consensus study. Lancet. 2005;366(9503):2112-2117. 
36. Pisani MA, Inouye SK, McNicoll L, Redlich CA. Screening for preexisting cognitive impairment in older intensive care unit patients: use of proxy assessment. J Am Geriatr Soc. 2003;51(5):689-693.

37. Braes T, Milisen K, Foreman MD. Assessing cognitive function. In: Capezuti E, Zwicker D, Mezey M, Fulmer T, editors. Evidence-based Geriatric Nursing Protocols for Best Practice. 3rd ed. New York, NY: Springer; 2008:41-56.

38. American Psychiatric Association. Delirium, dementia, and amnestic and other cognitive disorders. In: American Psychiatric Association, editor. DSM-IV-TR: Diagnostic and Statistical Manual of Mental Disorders. 4th ed. Washington, DC: American Psychiatric Association; 2000:147-171.

39. Foreman MD, Fletcher K, Mion LC, Simon L. Assessing cognitive function. Geriatr Nurs. 1996;17(5):228-232.

40. Jorm AF. A short form of the Informant Questionnaire on Cognitive Decline in the Elderly (IQCODE): Development and cross-validation. Psychol Med. 1994;24(1):145-153.

41. McDougall GJ. A review of screening instruments for assessing cognition and mental status in older adults. Nurse Pract. 1990;15(11):18-28.

42. Carolan Doerflinger DM. How to try this: the mini-cog. Am J Nurs. 2007;107(12):62-72.

43. Borson S, Brush M, Gil E, et al. The Clock Drawing Test: utility for dementia detection in multiethnic elders. J Gerontol A Biol Sci Med Sci. 1999;54(11):M534-M540.

44. Borson S, Scanlan J, Brush M, Vitaliano P, Dokmak A. The mini-cog: a cognitive 'vital signs' measure for dementia screening in multi-lingual elderly. Int J Geriatr Psychiatry. 2000;15(11):1021-1027.

45. Borson S, Scanlan JM, Chen P, Ganguli M. The Mini-Cog as a screen for dementia: Validation in a population-based sample. J Am Geriatr Soc. 2003;51(10):1451-1454.

46. Borson S, Scanlan JM, Watanabe J, Tu SP, Lessig M. Simplifying detection of cognitive impairment: comparison of the mini-cog and mini-mental state examination in a multiethnic sample. J Am Geriatr Soc. 2005;53(5):871-874.

47. Lorentz WJ, Scanlan JM, Borson S. Brief screening tests for dementia. Can J Psychiatry. 2002;47(8):723-733.

48. Lyons DL, Grimley SM, Sydnor L. Double trouble: when delirium complicates dementia. Nursing. 2008;38(9):48-54.

49. Scanlan J, Borson S. The Mini-Cog: receiver operating characteristics with expert and naive raters. Int J Geriatr Psychiatry. 2001;16(2): 216-222.

50. Voyer P, Cole MG, McCusker J, Belzile E. Prevalence and symptoms of delirium superimposed on dementia. Clin Nurs Res. 2006;15(1): 46-66.

51. Hoogerduijn JG, Schuurmans MJ, Duijnstee MS, de Rooij SE, Grypdonck MF. A systematic review of predictors and screening instruments to identify older hospitalized patients at risk for functional decline. J Clin Nurs. 2007;16(1):46-57.

52. Bradway C, Hirschman KB. Working with families of hospitalized older adults with dementia: caregivers are useful resources and should be part of the care team. Am J Nurs. 2008;108(10):52-60.

53. Carpenito-Moyet LJ. Chronic confusion. In: Carpenito-Moyet LJ, editor. Nursing Diagnosis: Application to Clinical Practice. Philadelphia, PA: Wolters Kluwer/Lippincott Williams \& Wilkins; 2010:175-180.

54. Hall GR, Maslow K. Working with families of hospitalized older adults with dementia. Am J Nurs. 2008;108(10):57-58.

55. Hilgers J. Comforting a confused patient: Learn how simple interventions and diversions can a help a confused patient. Nursing. 2003; $33(1): 48-50$.
56. Miller CA. Communication difficulties in hospitalized older adults with dementia. Am J Nurs. 2008;108(3):58-66.

57. Moyle W, Olorenshaw R, Wallis M, Borbasi S. Best practice for the management of older people with dementia in the acute care setting: a review of the literature. Int J Older People Nurs. 2008;3(2):121-130.

58. Smith M, Hall GR, Gerdner L, Buckwalter KC. Application of the progressively lowered stress threshold model across the continuum of care. Nurs Clin North Am. 2006;41(1):57-81.

59. Yuhas N, McGowan B, Fontaine T, Czech J, Gambrell-Jones J. Psychosocial interventions for disruptive symptoms of dementia. J Psychosoc Nurs Ment Health Serv. 2006;44(11):34-42.

60. Algase, D. Wandering. In: Ackley BJ, Ladwig GB, editors. Nursing Diagnosis Handbook: A Guide to Planning Care. 7th ed. St Louis: Mosby Elsevier; 2006:1315-1323.

61. Allen LA. Treating agitation without drugs. Am J Nurs. 1999;99(4): $36-42$.

62. Cunningham $\mathrm{C}$. Understanding challenging behaviour in patients with dementia. Nurs Stand. 2006;20(47):42-45.

63. Evans LK, Cotter VT. Avoiding restraints in patients with dementia: understanding, prevention, and management are the keys. Am J Nurs. 2008;108(3):40-49.

64. Jones J, Borbasi S, Nankivell A, Lockwood C. Dementia related aggression in the acute sector: is a Code Black really the answer? Contemp Nurse. 2006;21(1):103-115.

65. McCloskey RM. Caring for patients with dementia in an acute care environment. Geriatr Nurs. 2004;25(3):139-144.

66. McGonigal-Kenny ML, Schutte DL. Nonpharmacologic management of agitated behaviors in persons with Alzheimer disease and other chronic dementing conditions. J Gerontol Nurs. 2006;32(2):9-14.

67. Rader J, Barrick AL, Hoeffer B, et al. The bathing of older adults with dementia: easing the unnecessarily unpleasant aspects of assisted bathing. Am J Nurs. 2006;106(4):40-48.

68. Rowe M. Wandering in hospitalized older adults: identifying risk is the first step in this approach to preventing wandering in patients with dementia. Am J Nurs. 2008;108(10):62-70.

69. Wheeler SL, Houston $\mathrm{K}$. The role of diversional activities in the general medical hospital setting. Holist Nurs Pract. 2005;19(2):87-89.

70. Ouldred E, Bryant C. Dementia care. Part 2: understanding and managing behavioral challenges. Br J Nurs. 2008;17(4):242-247.

71. Ouldred E, Bryant C. Dementia care. Part 1: guidance and the assessment process. Br J Nurs. 2008;17(3):138-145.

72. Holden U. Dementia in acute units: Confusion. Nurs Stand. 1995; 9(17):37-39.

73. Christensen MD, White HK. Dementia assessment and management. J Am Med Dir Assoc. 2006;7(2):109-118.

74. Milisen K, Braes T, Fick DM, Foreman MD. Cognitive assessment and differentiating the 3 Ds (dementia, depression, delirium). Nurs Clin North Am. 2006;41(1):1-22.

75. Waldemar G, Dubois B, Emre M, et al; EFNS. Recommendations for the diagnosis and management of Alzheimer's disease and other disorders associated with dementia: EFNS guideline. Eur J Neurol. 2007;14(1):e1-e26.

76. Folstein MF, Folstein SE, McHugh PR. "Mini-mental state". A practical method for grading the cognitive state of patients for the clinician. J Psychiatr Res. 1975;12(3):189-198. 
Nursing: Research and Reviews

\section{Publish your work in this journal}

Nursing: Research and Reviews is an international, peer-reviewed, open access journal publishing original research, reports, reviews and commentaries on all aspects of nursing and patient care. These include patient education and counselling, ethics, management and organizational issues, diagnostics and prescribing, economics and

resource management, health outcomes, and improving patient safety in all settings. The manuscript management system is completely online and includes a very quick and fair peer-review system. Visit http://www.dovepress.com/testimonials.php to read real quotes from published authors.

Submit your manuscript here: http://www.dovepress.com/nursing-research-and-reviews-journal 